\title{
COST EFFICIENT MULTI-SOURCE ENERGY MIXING FOR RENEWABLE ENERGY MICROGRIDS USING HYBRID ABC- PSO ALGORITHM
}

\author{
Ebru Kömürgöz, \\ Cemal Keleş*
}

Department of Electrical-Electronics, Engineering, Inonu University, Malatya, Turkey

\begin{abstract}
:
Energy management systems can provide a variety of features and services to prosumers. One of its most important functions is to determine cost-effective energy mixing rates by evaluating the unit price and power of energy resources. This study mainly proposes a hybrid optimization based on two heuristic algorithms: Artificial Bee Colony (ABC) and Particle Swarm Optimization (PSO) algorithms. A hybrid ABC-PSO algorithm has been applied to solve the energy efficient multi-source energy mixing problem in Matlab. The proposed algorithm has been tested in the simulation of an energy management system including a grid, solar panel, wind turbine, and storage unit. The results show that the proposed algorithm responds appropriately to meet the hourly changing demand of the consumer in cases of energy production fluctuations in renewable energy sources and dynamic electricity price tariff implementation of the grid. This method can provide cost efficiency by maintaining the energy balance of consumers in smart grids. The algorithm has a simple structure, thus the method provides a solution for low-cost energy management applications in the microgrid.
\end{abstract}

Keywords:

Smart grid, hybrid ABC-PSO algorithm, cost efficient energy mixing, microgrid.

\section{INTRODUCTION}

Global warming, limited energy resources, increasing dependence on energy require modernization of conventional grid architecture, innovative solutions and technologies [1]. In this context, the energy industry is transitioning to a new era called "smart energy". Energy will become more sustainable, reliable and green by using information and communication technology in the near future. On the other hand, the smart energy system is a cost effective system that incorporates renewable energy sources into the process. Smart grid, demand response, microgrid, smart home are the main concepts in this field [2]. The negative effects of traditional fossil fuels on the environment and the public awareness of developments in renewable energy technology have increased the interest in microgrids [3]. Microgrids can be described as small, local distribution systems containing micro resources such as solar panels, wind turbines, storage systems, micro turbines, fuel cells [1]. 
Renewable energy sources have an intermittent generation due to their nature. This situation considerably fluctuates the demands of the consumers [4]. The microgrid allows users to decide on setting the time and amount of consumption [2]. Generally, demand-side management in microgrids can be carried out using a smart optimization process [5].

The use of renewable energy sources in the energy systems of buildings has a significant impact [6]. The total energy consumption of buildings corresponds to $40 \%$ of the world's energy consumption. It is thought that in the close future, buildings will be able to produce their consumed energy. This means zero energy buildings. Since fossil fuels are unsustainable, the idea of using renewable energy in smart homes has generated great interest from the public. With the advance of home energy management systems (HEMS) in smart grids, users have the opportunity to actively participate in demand-side management (DSM). Through the smart home energy management system, users can optimize their electricity consumption and improve their comfort levels [7]. Figure 1 shows five technical functional diagrams of energy efficiency in smart homes [8].

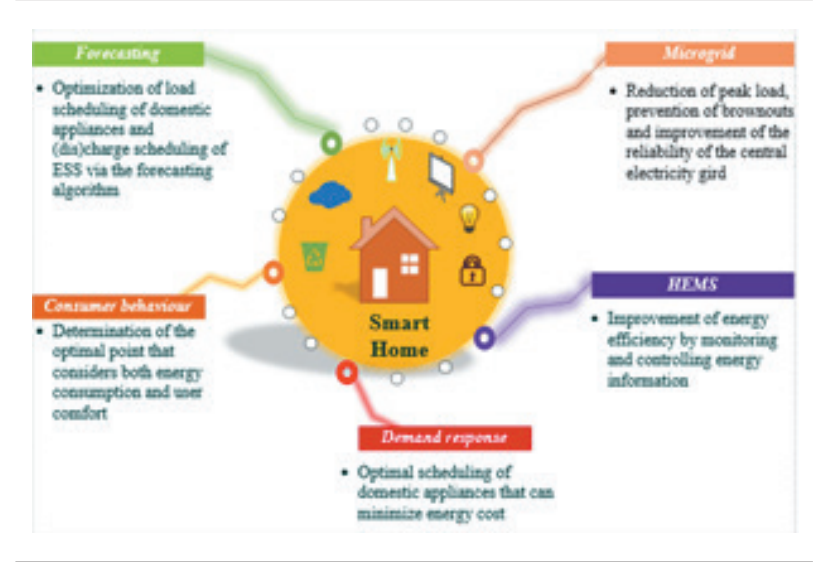

Figure 1 - Conceptual diagram of energy efficiency in smart homes [8].

Smart consumers are the end user members of the future smart grid and play a key role in the supply-demand balance [9]. With the active participation of consumers in demand management, the use of information and communication technology devices has become widespread in-home applications [10]. In the near future, it will be possible to optimize electricity consumption, meet supply constraints and minimize costs in smart homes with energy management systems. At the same time, it is usual to predict that the continuity of the desired comfort level for the users will be facilitated by energy management systems [11]. Automatic Home Energy Management System (AHEMS) with appropriate algorithms in a smart home should allow optimization of the existing energy system (grid, loads, local generation and storage unit) at time-varying tariffs. In this context, algorithms in the core of AHEMS allow flexible use of some end-user loads. Thus, it contributes to taking advantage of higher levels of renewable energy generation and lowering the energy bills of consumers [12]. Data collected from smart measurement systems and meteorology can be updated by artificial intelligence techniques and machine learning algorithms to regulate the relationships between various variables such as energy consumption, radiation, temperature, time, and battery charge state [13]. Representation of artificial intelligence as "Energy Intelligence" on the subjects of cost, efficiency and energy monitoring is a guide for users [14].

There are various algorithms in the literature such as artificial bee colony $(\mathrm{ABC})$, particle swarm optimization (PSO), genetic algorithm, random search algorithm, differential evolution. Among these solution approaches, $\mathrm{ABC}[15]$ and PSO [16,17] are the most common techniques used to solve optimization problems [18]. Although the $\mathrm{ABC}$ algorithm has an effective discovery capability, it has weak exploitation capability. PSO has a good utilization ability. However, when the population or particle falls to the local minimum, the PSO has no ability to get out of the local minimum level. Therefore, PSO is not sufficient for the exploration of the search space [19]. Most of the time, a hybrid optimization method combining two or more methodologies is proposed to increase the convergence time of the optimization process [20]. This hybrid optimization process is carried out by improving PSO's weak discovery ability and $\mathrm{ABC}$ 's weak exploitation ability [21].

This study proposes a hybrid optimization based on two heuristic algorithms: $\mathrm{ABC}$ and PSO. The hybrid ABC-PSO algorithm has been applied in Matlab to solve the energy efficient multi-source energy mixing problem. The proposed algorithm has been tested in a simulation of an energy management system including grid, solar panel, wind turbine and battery. The results show that the proposed algorithm responds appropriately to meet the hourly changing demand of the consumer. The production fluctuations of renewable energy resources and the dynamic electricity price tariff of the grid were taken into account in this process. This method can provide cost efficiency by maintaining the energy balance of consumers in smart grids. The structural simplicity of the method provides a solution for low cost, smart and local energy management applications. 


\section{METHODOLOGY}

\subsection{THE PROBLEM OF ENERGY MIXING IN MICROGRIDS INCLUDING RENEWABLE ENERGY SOURCES}

The architectural structure of the microgrid energy management system consists of renewable energy sources, grid, battery, loads, smart algorithm and energy mixing components as shown in Figure 2.

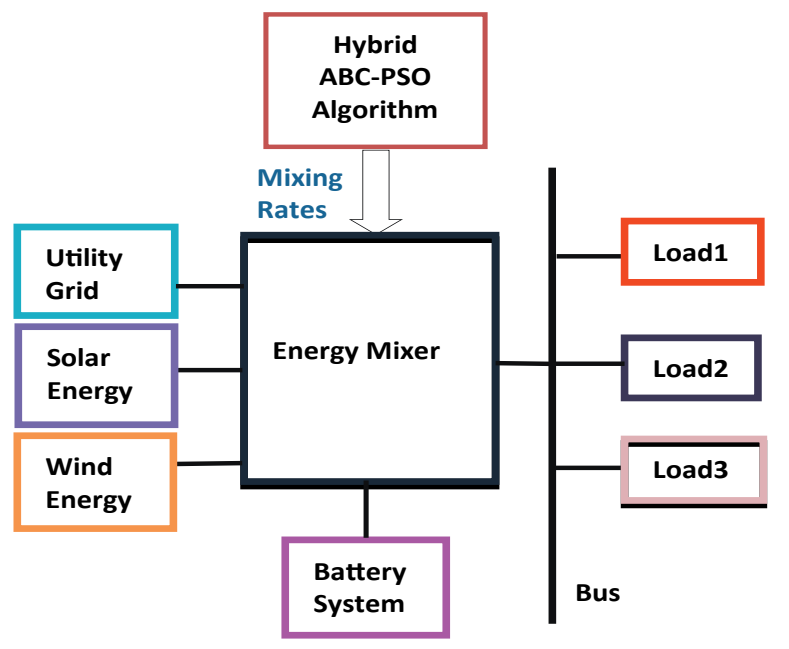

Figure 2 - The architectural structure of the optimal microgrid management system [22].

In the authors' previous studies, random search optimization (RSO) [22] and particle swarm optimization (PSO) algorithms [16] were applied to find cost-effective optimal energy mixing ratios according to the power levels and unit energy costs of renewable energy sources. In this study, the hybrid ABC-PSO algorithm was applied to solve the energy efficient multi-source energy mixing problem. The determination of energy mixture rates was carried out in Matlab. Suppose that the energies of solar panels, wind turbines, battery and grid are combined at mixing rates expressed as $\alpha_{s}$ for solar, $\alpha_{w}$ for wind, $\alpha_{b}$ for the battery system, and $\alpha_{g}$ for grid. In this case, the total energy sent from sources to grid loads can be expressed as follows [22]:

$$
E_{T}=\alpha_{s} E_{s}+\alpha_{w} E_{w}+\alpha_{b} E_{b}+\alpha_{g} E_{g}
$$

Equation 1 - Total energy from sources.

In here, $E_{3} E_{w}, E_{b}, E_{g}$ represent instantaneous energies from the sun, wind, battery and grid, respectively. Considering the unit energy costs of $c_{s}$ for the solar system, $c_{w}$ for the wind system, $c_{b}$ for the battery system and $c_{g}$ for the grid, the cost function to be used in optimization for energy efficient, cost effective mixing of resources can be written as follows [22]:

$$
F=\alpha_{s} c_{s} E_{s}+\alpha_{w} c_{w} E_{w}+\alpha_{b} c_{b} E_{b}+\alpha_{g} c_{g} E_{g}
$$

Equation 2 - Cost function.

Let consider that the mixing rate coefficients are updated at every $\Delta t$ time interval by the hybrid ABCPSO optimization algorithm. Thus, the cost function minimized by using the average output power of the sources in $\Delta t$ time intervals can be written as [16],

$$
F(\alpha)=\alpha_{s} c_{s} P_{s} \Delta t+\alpha_{w} c_{w} P_{w} \Delta t+\alpha_{b} c_{b} P_{b} \Delta t+\alpha_{g} c_{g} P_{g} \Delta t
$$

Equation 3 - Minimized cost function.

where $P_{s} P_{w}$ and $P_{b}$ express the average output power of the solar, wind and battery system, respectively and $P_{g}$ shows the average power drawn from the grid. Also, the mixing rate vector for the microgrid is shown as $\alpha=\left[\alpha_{s} \alpha_{w} \alpha_{b} \alpha_{g}\right]$. It is important to balance energy production $\left(E_{T}\right)$ and energy demand $\left(E_{D}\right)$ in the microgrid. Energy supply and demand balance status in the microgrid can be written as [16],

$$
\gamma P_{D}=P_{T}=\alpha_{s} P_{s}+\alpha_{w} P_{w}+\alpha_{b} P_{b}+\alpha_{g} P_{g}
$$

Equation 4 - Energy balance status.

where $P_{T}$ and $P_{D}$ indicate the total average output power of the sources and the total demand power, respectively. The case of $y>1$ provides a virtual demand to reduce energy shortage by allowing some over-production. Equation 5 can be written to determine the energy balance error [16].

$$
E_{h}=P_{D} \Delta t-P_{T} \Delta t
$$

Equation 5 - Energy balance error.

Considering the energy balance boundary condition expressed in Equation 4, the mixing ratio of the grid can be calculated with the following equation [16]:

$$
\alpha_{g}=\left(\gamma P_{D}-\alpha_{s} P_{s}-\alpha_{w} P_{w}-\alpha_{b} P_{b}\right) / P_{g}
$$

Equation 6 - Mixing ratio of the grid. 
$\alpha_{g} \leq 0$ condition indicates the island-mode operation of the microgrid. According to $\alpha_{g}$ value, the cost function has been calculated as Equation 7 to determine the working status of the system in islanded mode and to maintain the working status of grid-connected [16]:

$$
F(a)=\left\{\begin{array}{c}
a_{s} c_{s} P_{s} \Delta t+a_{w} c_{w} P_{w} \Delta t+a_{b} c_{b} P_{b} \Delta t \\
+a_{g} c_{g} P_{g} \Delta t a_{g}>0 \\
F_{h} a_{g} \leq 0
\end{array}\right.
$$

Equation 7 - Calculated cost function.

where $F_{h}$ means high cost value to avoid the islanded mode operation of the microgrid. Therefore, the hybrid ABC-PSO algorithm does not allow the system to run in islanded mode [16].

\subsection{APPLICATION OF HYBRID ABC-PSO ALGORITHM FOR ENERGY MIXING RATE OPTIMIZATION}

Discovery and utilization capabilities of populationbased algorithms are among the main features of these algorithms. The discovery searches unknown regions to find the global optimum, while utilization tries to apply knowledge of previous solutions to find better solutions.

To achieve the best result, discovery and utilization abilities should be balanced. However, researches show that $\mathrm{ABC}$ algorithm performs good discovery and weak utilization in the solution search process [23]. PSO has good utilization ability. On the other hand, when the population or particle drops to the local minimum, the PSO cannot get out of the local minimum level. In addition, the PSO is not sufficient for the discovery of the search space [19]. In order to take advantage of these two algorithms and eliminate their disadvantages, a hybrid global optimization approach called ABC-PSO can be used [24].

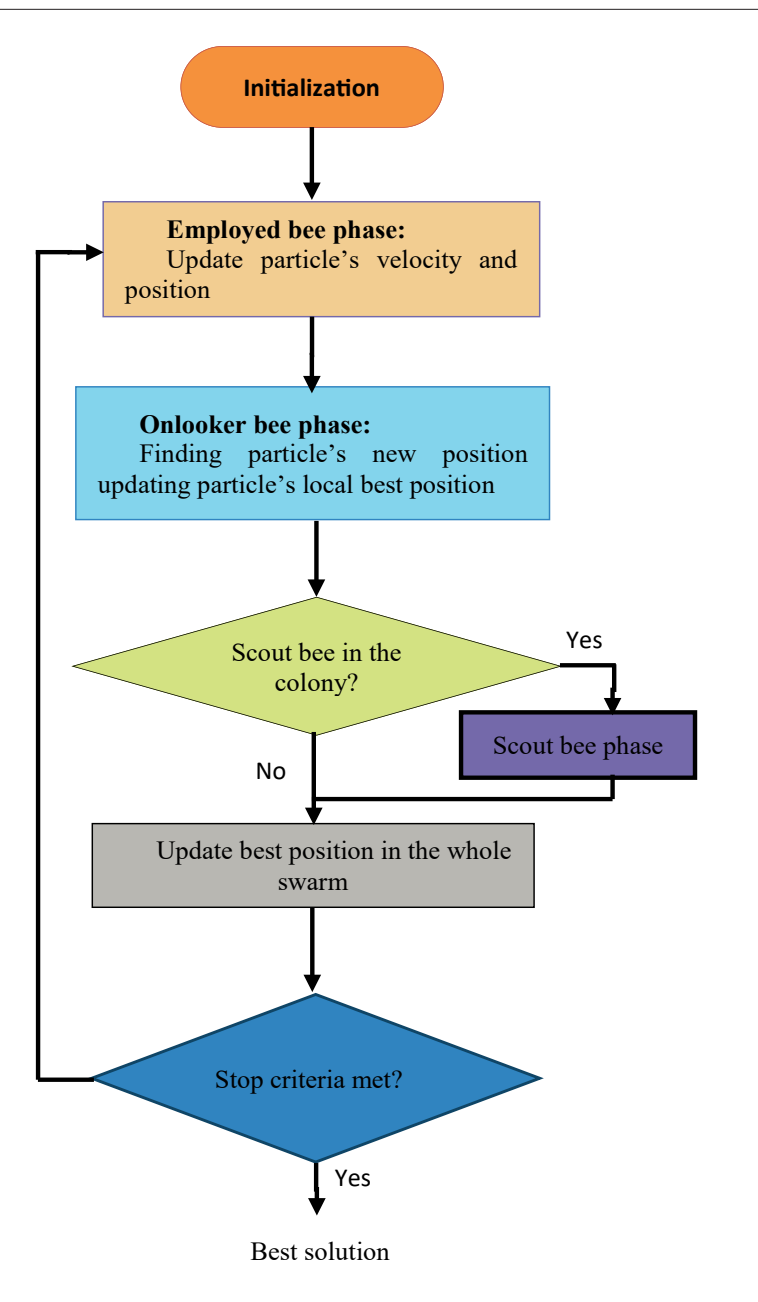

Figure 3 - Flow chart of the proposed algorithm[24].

Figure 3 shows the flow chart of the proposed optimization algorithm. In this algorithm, three phases of $\mathrm{ABC}$ are used. For the employed bee phase, PSO's method and speed of finding new food sources are used. After the location of the new food source is updated, the best available position is determined using Equation 8 [24].

$$
\text { Pbest }_{i}^{t+1}=\left\{\begin{array}{c}
\text { Pbest }_{i}^{t}, \text { if } f\left(\text { Pbest }_{i}^{t}\right) \leq f\left(X_{i}^{t+1}\right) \\
X_{i}^{t+1}, \text { if }\left(\text { Pbest }_{i}^{t}\right)>f\left(X_{i}^{t+1}\right)
\end{array}\right.
$$

Equation 8 - Best available position.

At the onlooker bee phase determined by the employed bee, onlooker bees will find and memorize a new food source location based on the knowledge of the best food source location visited by the employed bee. This operation is performed according to Equation 9 [24]. 


$$
X_{i j}= \begin{cases}\text { Pbest }_{\mathrm{ij}} & \text {, if } j \neq m \\ \text { Pbest }_{\mathrm{im}}+\varnothing_{\mathrm{i} m}\left(\text { Pbest }_{\mathrm{im}}-\text { Pbest }_{\mathrm{km}}\right) & \text {, if } j=m\end{cases}
$$

Equation 9 - Food source location.

where $m \in[1, D]$ is chosen randomly, $k \neq j$ and $\varnothing_{i m}$ are random numbers generated between -1 and 1 . The scout bee phase is similar as in the $\mathrm{ABC}$ algorithm [24].

In order to carry out the energy mixing rates to the energy mixer block, the normalization should be performed to satisfy $\alpha_{s}+\alpha_{w}+\alpha_{b}+\alpha_{g}=1$ condition. Equation 10 is used for this process.

$$
\tilde{a}_{x}=\frac{a_{x}}{\sum_{i=\{s, w, b\}}^{4} a_{i}}, x=\{s, w, b, g\}
$$

Equation 10 - Normalization of mixing rates.

\section{SIMULATION STUDY FOR ENERGY MANAGEMENT IN MICROGRIDS INCLUDING RENEWABLE ENERGY RESOURCES}

In this section, a simulation study was carried out to perform the hybrid ABC-PSO algorithm for cost-effective renewable energy mixing in a home energy management system shown in Figure 4. The simulation model consists of four energy systems: solar, wind, battery with an output power of $1 \mathrm{~kW}$ and electricity grid with power limitation of $2 \mathrm{~kW}[16]$.

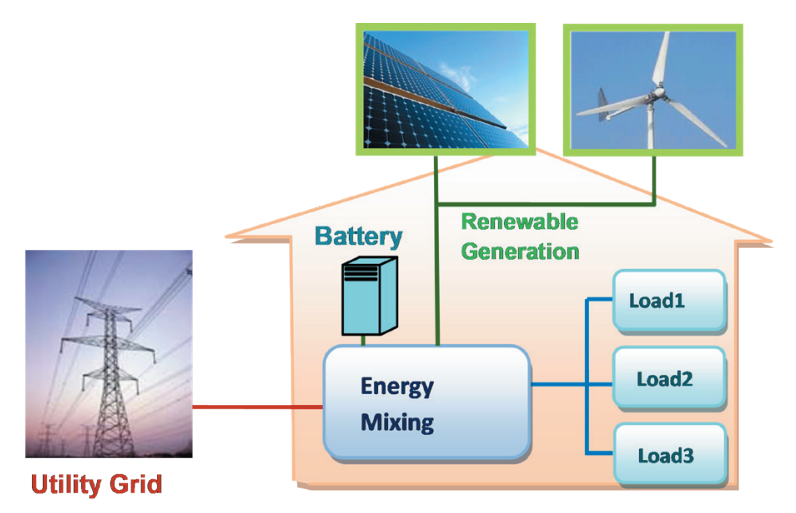

Figure 4 - Simulation model for cost effective energy management in the microgrid [16].

In the simulation study, hourly generation profiles of solar and wind energy systems shown in Figure 5 were used.
The average demand profile shown in Figure 6 was used for the demand profile of the home considered as a microgrid.

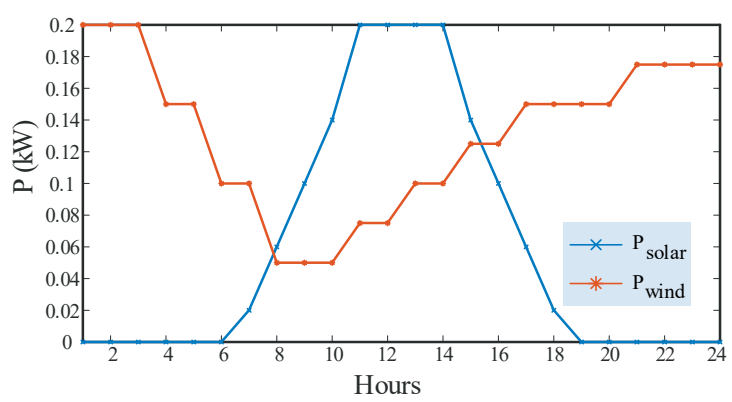

Figure 5 - Production profile of the solar and wind energy systems [16].

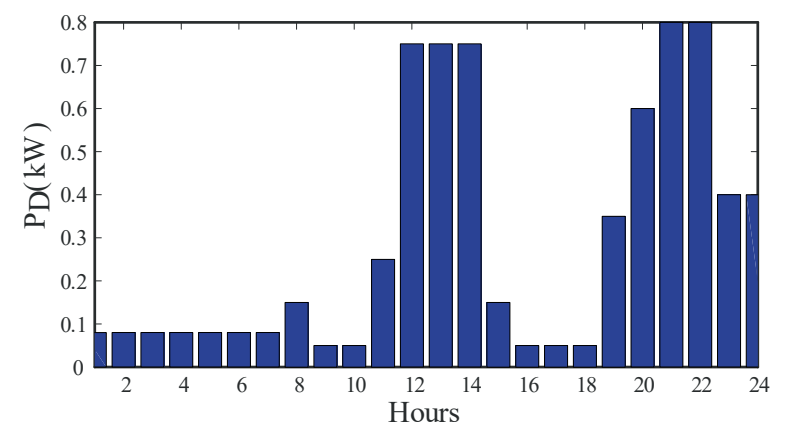

Figure 6 - Average demand profile of the home.

It is accepted that the energy unit costs of the solar, wind and storage system used in the simulation and the energy unit price of the grid applied in the dynamic tariff are determined as in Figure 7 [16].

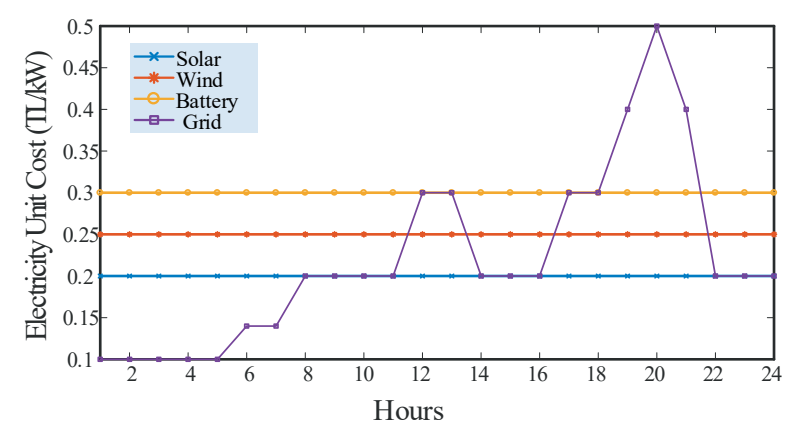

Figure 7 - Pricing profile of energy resources. 


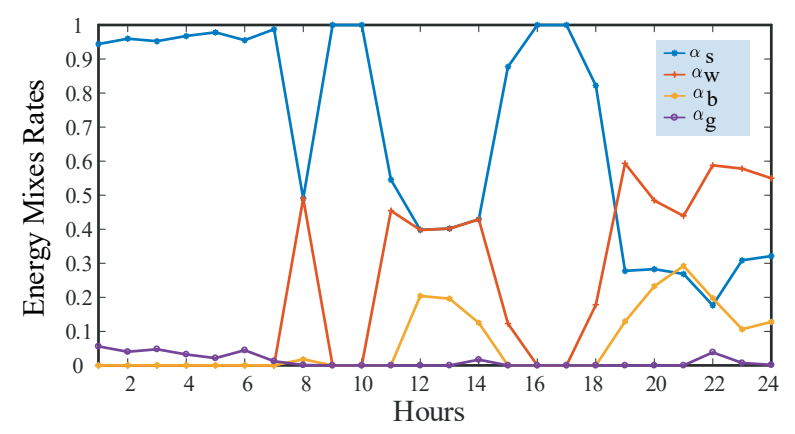

Figure 8 shows the hourly calculated mixing rates of the energy sources by the hybrid ABC-PSO algorithm.

The energy balance error given in Figure 9 shows that the proposed algorithm within the scope of the production fluctuations of solar and wind energy sources and the dynamic energy price tariff of the grid can maintain the supply-demand balance. Figure 10 shows the hourly changes of the energy efficient cost function. It is seen from this figure that the hybrid ABC-PSO algorithm achieves better results than $\mathrm{ABC}$. It can be said that this is achieved by combining $\mathrm{ABC}$ and $\mathrm{PSO}$ algorithms.

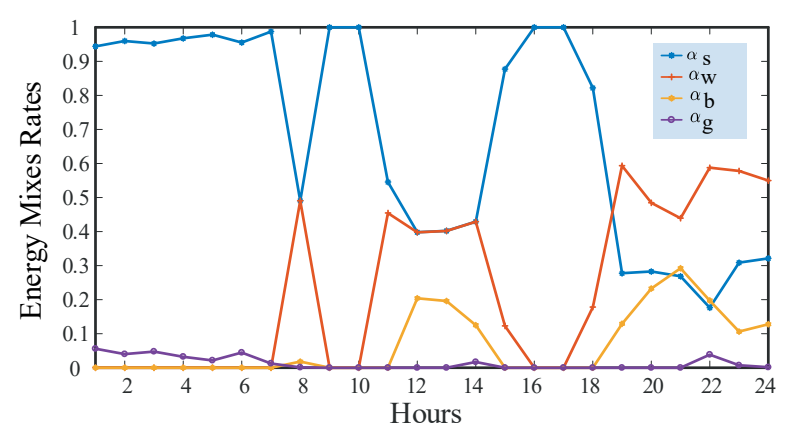

Figure 8 - Energy mixing rates determined by the proposed hybrid ABC-PSO algorithm.

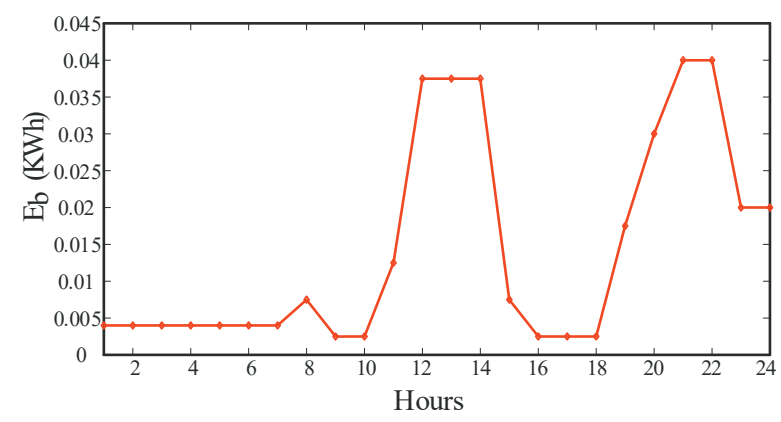

Figure 9 - Energy balance error of microgrid.

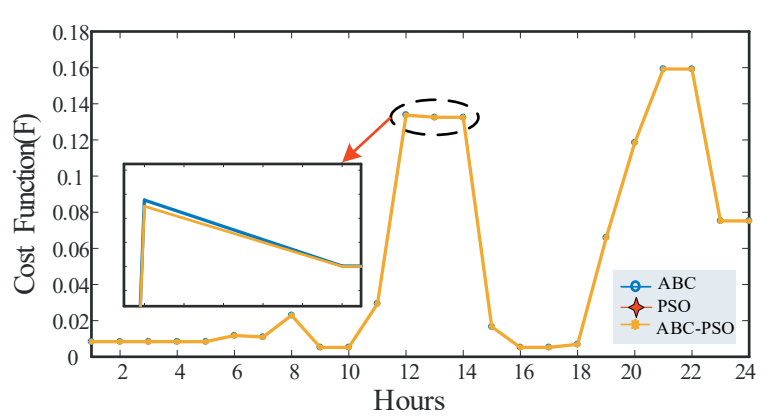

Figure 10 - Hourly change of cost function.

\section{CONCLUSION}

This paper demonstrates the use of the hybrid ABCPSO algorithm to solve the energy efficient, cost-effective multisource energy mixing problem in microgrids containing renewable energy sources. Our numerical analyzes show that the proposed hybrid ABC-PSO algorithm responds appropriately to meet the hourly changing demand of the consumer in case of power generation fluctuations in renewable energy sources and dynamic electricity price tariffs in the grid. By preserving the energy balance and without affecting the life comfort of the users, the cost efficiency can be achieved through the optimization algorithm by taking into account the dynamic electricity tariffs.

The method has a simple algorithm; therefore, it offers a solution opportunity for low cost, smart and local energy management applications.

\section{REFERENCES}

[1] Y. Yoldaş, A. Önen, S. M. Muyeen, A. V. Vasilakos, and İ. Alan, 'Enhancing smart grid with microgrids: Challenges and opportunities', Renew. Sustain. Energy Rev., vol. 72, pp. 205-214, May 2017.

[2] H. Merdanoğlu, 'Scheduling for home energy management system', Middle East Technical University, 2018.

[3] M.E. Şeker, 'Cost study of microgrid application in Turkey', Ankara Yıldırım Beyazıt University, 2020.

[4] Y. Xu, C. Yan, H. Liu, J. Wang, Z. Yang, and Y. Jiang, 'Smart energy systems: A critical review on design and operation optimization', Sustain. Cities Soc., vol. 62, no. 200, pp. 102-369, Nov. 2020. 
[5] A. M. A. Haidar, A. Fakhar, and A. Helwig, 'Sustainable energy planning for cost minimization of autonomous hybrid microgrid using combined multiobjective optimization algorithm', Sustain. Cities Soc., vol. 62, no. December 2019, pp. 102-391, Nov. 2020.

[6] S. Longo, F. Montana, and E. Riva Sanseverino, 'A review on optimization and cost-optimal methodologies in low-energy buildings design and environmental considerations', Sustain. Cities Soc., vol. 45, pp. 87-104, Feb. 2019.

[7] X. Kong, B. Sun, D. Kong, and B. Li, 'Home energy management optimization method considering potential risk cost', Sustain. Cities Soc., vol. 62, pp. 102-378, Nov. 2020.

[8] H. Kim, H. Choi, H. Kang, J. An, S. Yeom, and T. Hong, 'A systematic review of the smart energy conservation system: From smart homes to sustainable smart cities', Renew. Sustain. Energy Rev., vol. 140, no. January, p. 110755, Apr. 2021.

[9] M. Boork, M. Thomtén, and M. Brolin, 'Key success factors and barriers to end user engagement in smart grid projects', BEHAVE2014 - Behav. Energy Effic. Conf., 2014.

[10] A. Molderink, V. Bakker, M. G. C. Bosman, J. L. Hurink, and G. J. M. Smit, 'Management and Control of Domestic Smart Grid Technology: Smart Grid, IEEE Transactions on', Smart Grid, IEEE Trans., vol. 1, no. 2, pp. 109-119, 2010.

[11] M. Vasirani and S. Ossowski, 'Smart consumer load balancing: state of the art and an empirical evaluation in the Spanish electricity market', Artif. Intell. Rev., vol. 39, no. 1, pp. 81-95, Jan. 2013.

[12] I. Gonçalves, Á. Gomes, and C. Henggeler Antunes, 'Optimizing the management of smart home energy resources under different power cost scenarios', Appl. Energy, vol. 242, pp. 351-363, May 2019.

[13] D. Kolokotsa, 'The role of smart grids in the building sector', Energy Build., vol. 116, pp. 703-708, Mar. 2016.

[14] D. A. Terciyanl1, 'Nesnelerin interneti ile enerji verimliliği uygulamalari', Elektr. Mühendisliği Derg., no. 467, pp. 26-28, 2021.

[15] W.-M. Lin, C.-S. Tu, and M.-T. Tsai, 'Energy Management Strategy for Microgrids by Using Enhanced Bee Colony Optimization', Energies, vol. 9, no. 1, p. 5, Dec. 2015.

[16] C. Keles, B. B. Alagoz, and A. Kaygusuz, 'Multisource energy mixing for renewable energy microgrids by particle swarm optimization', in 2017 International Artificial Intelligence and Data Processing Symposium (IDAP), 2017, pp. 1-5.

[17] M. Amer, A. Namaane, and N. K. M'Sirdi, 'Optimization of hybrid renewable energy systems (HRES) using PSO for cost reduction', in Energy Procedia, 2013, vol. 42, pp. 318-327.
[18] S. Singh, P. Chauhan, and N. J. Singh, 'Capacity optimization of grid connected solar/fuel cell energy system using hybrid ABC-PSO algorithm', Int. J. Hydrogen Energy, vol. 45, no. 16, pp. 10070-10088, 2020.

[19] M. S. Kiran and M. Gündüz, 'A recombinationbased hybridization of particle swarm optimization and artificial bee colony algorithm for continuous optimization problems', Appl. Soft Comput. J., vol. 13, no. 4, pp. 2188-2203, Apr. 2013.

[20] S. M. Zahraee, M. Khalaji Assadi, and R. Saidur, 'Application of Artificial Intelligence Methods for Hybrid Energy System Optimization', Renewable and Sustainable Energy Reviews, vol. 66. Elsevier, pp. 617-630, Dec-2016.

[21] Z. Li, W. Wang, Y. Yan, and Z. Li, 'PS-ABC: A hybrid algorithm based on particle swarm and artificial bee colony for high-dimensional optimization problems', Expert Syst. Appl., vol. 42, no. 22, pp. 8881-8895, Dec. 2015.

[22] C. Keles, B. Alagoz, A. Kaygusuz, and S. Alagoz, 'Cost efficient multi-source energy mixing for renewable energy microgrids by random search optimization', in International Artificial Intelligence and Data Processing Symposium (IDAP), 2016, pp. 29-33.

[23] H. Djellali, A. Djebbar, N. G. Zine, and N. Azizi, 'Hybrid Artificial Bees Colony and Particle Swarm on Feature Selection', in IFIP Advances in Information and Communication Technology, vol. 522, Springer International Publishing, 2018, pp. 93 105.

[24] T. T. Khuat and M. H. Le, 'A Novel Hybrid ABCPSO Algorithm for Effort Estimation of Software Projects Using Agile Methodologies', J. Intell. Syst., vol. 27, no. 3, pp. 489-506, Jul. 2018. 